\title{
El riesgo en la utilización de la cinta métrica torácica como método de pesaje bovino. Análisis estadístico
}

\author{
The risks in the use of the thoracic tape measure as a method of bovine weighing. \\ Statistical analysis
}

\author{
Vanessa Martínez Jiménez ${ }^{1}$, Josmar Mazucheli ${ }^{1}$, Isabele Picada Emanuelli ${ }^{2,3,4}$
}

\section{Resumen}

El objetivo del presente estudio fue evaluar si los métodos de pesaje mediante balanza digital (gold standard) e indirecto por medición torácica son concordantes aplicando el Coeficiente de Concordancia Lin (CCC), el Gráfico de Bland-Altman y la Curva de Supervivencia de la diferencia de las medias en bovinos Nelore. Se analizaron los datos de 46 novillos reportados en Watanabe et al. (2017) aplicando métodos alternativos. Se observó un peso medio mayor en las medidas obtenidas con balanza digital, mientras que el rango obtenido con la cinta torácica fue mayor, con una diferencia media entre los pesos de $13.8 \mathrm{~kg}$. Además, el peso obtenido con la balanza digital presenta un mínimo y mediana mayor que el obtenido mediante la cinta torácica. Se comprobó con los tres métodos que las mediciones con la balanza digital y la cinta torácica no son concordantes para los datos del peso de novillos Nelore. Las medidas presentan diferencias que imposibilitan que estos métodos sean intercambiables; por lo tanto, el cálculo del peso de bovinos Nelore mediante la cinta torácica proporciona medidas que pueden llevar a conclusiones erróneas.

Palabras clave: coeficiente de concordancia de Lin; curva de supervivencia; gráfico de Bland-Altman; pesaje indirecto

\section{Abstract}

The aim of this study was to evaluate if the methods of weighing Nellore cattle by digital scale (gold standard) and indirect by thoracic measurement are concordant applying the Lin's Concordance Correlation Coefficient (CCC), the Bland-Altman Graph and the Survival Curve of the difference of the mean. The data of 46 steers reported in Watanabe

\footnotetext{
${ }^{1}$ Universidade Estadual de Maringá/UEM, Maringá, Brasil

${ }^{2}$ Centro Universitário de Maringá/UniCesumar/ICETI, Maringá, Brasil

${ }^{3}$ Instituto Cesumar de Ciência, Tecnologia e Inovação/ICETI Maringá, Brasil

${ }^{4}$ E-mail: isabelevet@hotmail.com
}

Recibido: 15 de marzo de 2018

Aceptado para publicación: 6 de diciembre de 2018 
et al. (2017) was analyzed applying these alternative methods. A greater average weight was observed in the measurements obtained with the digital scale, while the range obtained with the thoracic tape was greater, with an average difference between weights of $13.8 \mathrm{~kg}$. In addition, the weight obtained with the digital balance has a minimum and median greater than that obtained by the thoracic tape. It was verified with the three methods that the measurements with the digital balance and the thoracic tape are not concordant for the weight data of Nelore steers. The measures present differences that make it impossible for these methods to be interchangeable, therefore, the calculation of the weight of Nelore cattle through the thoracic tape provides measures that can lead to erroneous conclusions.

Key words: Bland-Altman's graph; indirect weighing; Lin's concordance correlation coefficient; survival curve

\section{INTRODUCCIÓN}

Brasil fue el segundo país en el ranking mundial de exportación de carne bovina y el segundo productor mundial en 2016, escasamente por detrás de la India, según el Departamento de Agricultura de Estados Unidos (USDA, 2017), lo cual resalta la importancia del sector bovino en la economía brasileña y la necesidad patente de metodologías estandarizadas para la valoración del animal vivo, la carne y sus subproductos. Entre estas, se tiene la necesidad de conocer el peso de los animales, toda vez que el peso es un indicativo del rendimiento productivo, del estado de salud y sirve de ayuda para el manejo animal y para la selección genética (Garro y Rosales, 1996; Mahecha et al., 2002; Reis et al., 2008).

El gold standard para el pesaje de animales bovinos es la balanza digital por su alta precisión; sin embargo, el costo es una limitante para muchos ganaderos y su empleo puede, en algunos casos causar estrés al animal, especialmente si es que tiene que recorrer largas distancias hasta llegar a los corrales de pesaje (Heinrichs et al., 1992). Debido a esto, el pesaje indirecto por medio de la cinta torácica es ampliamente utilizado. Este método consiste en utilizar una cinta métrica colocada en torno al tórax del animal para, posteriormente, calcular el peso con base a ecuaciones previamente validadas (Heinrichs y Hargrove, 1987; Setim et al., 2010).

Según Silva et al. (2006), el peso del animal vivo y la medida torácica están altamente correlacionadas; no obstante, Setim et al. (2010) indican que pueden existir diferencias para el bovino entre $8 \mathrm{~kg}$ por encima y $4.5 \mathrm{~kg}$ por debajo con el uso de la cinta torácica. De igual modo, Mejia et al. (1996) encontraron un error máximo de $15 \mathrm{~kg}$ en animales con un peso inferior a $175 \mathrm{~kg}$ y de hasta $40 \mathrm{~kg}$ en animales de mayor peso. Para poder corregir estas deficiencias se han desarrollado modelos de predicción, cuya bondad de ajuste depende de factores como la raza, el número de animales utilizados en la construcción del modelo y el peso del animal. De esta forma, utilizando como medida de ajuste el error cuadrático medio (ECM), pueden citarse los modelos de Ozkaya y Bozkurt (2009) con un ECM de 69.25, Yan et al. (2009) con 59.66, Kashoma et al. (2011) con 38.50 y Lesosky et al. (2013) con 28.86.

Por otro lado, se han hecho estudios para analizar la concordancia entre métodos de pesaje, entre los cuales pueden citarse el de Cruz et al. (2003), que comprobaron el uso del peso total de los alimentos en guarderías como medida indirecta para el cálculo del 
consumo individual utilizando el Coeficiente de Concordancia de Lin y el gráfico de BlandAltman, donde concluyen que las dos herramientas evaluadas presentaban diferencias significativas. Ante estas inconsistencias surge la necesidad de evaluar si las metodologías de pesaje, gold standard, e indirecta por medición torácica, son concordantes y, por tanto, pueden ser intercambiables. Es así, que el presente estudio tuvo como objetivo evaluar la concordancia de estas dos metodologías aplicando tres modelos estadísticos: el Coeficiente de Correlación de Concordancia de Lin (CCC), el Gráfico de BlandAltman y la Curva de Supervivencia de la diferencia de las medias en novillos Nelore.

\section{Materiales y Métodos}

La base metodológica de esta investigación fue un análisis secundario de la base de datos completa disponible en Watanabe et al. (2017). La base de datos contenía información referente a 46 bovinos macho Nelore con una edad media entre 15 y 17 meses, criados sobre manejo semiextensivo. Las variables estudiadas en los animales fueron el peso vivo medido con la balanza electrónica digital Beckhauser ${ }^{\circledR}$ y el peso calculado a partir de las medidas realizadas con la cinta torácica Zolla ${ }^{\circledR}$, que fueron tomadas de caudal al omóplato pasando por el esternón y por las apófisis espinosas de las vértebras torácicas (Watanabe et al., 2017).

Estos datos volvieron a ser analizados mediante el Coeficiente de Correlación de Concordancia de Lin (CCC), el Gráfico de Bland-Altman y la Curva de Supervivencia de la diferencia de las medias.

El coeficiente de Lin puede variar entre -1 y 1 y su valor absoluto no puede ser mayor que el coeficiente de correlación de Pearson. La escala descriptiva (Cuadro 1) para los posibles valores del CCC en el caso de va-
Cuadro 1. Nivel de concordancia según el valor del Coeficiente de Concordancia de Lin en variables continuas

\begin{tabular}{ll}
\hline Valor de $p_{c}$ & Nivel de concordancia \\
\hline$<0.90$ & Pobre \\
$0.90-0.95$ & Moderada \\
$0.95-0.99$ & Considerable \\
$>0.99$ & Casi perfecta \\
\hline
\end{tabular}

riables continuas, propuesta por McBride (2005), se muestra en el Cuadro 1.

El gráfico de concordancia de BlandAltman describe la concordancia entre dos variables cuantitativas construyendo los límites de concordancia, los cuales se calculan utilizando la media y la desviación típica $(s)$ de las diferencias entre las dos medidas (Bland y Altman, 1999). El método calcula la diferencia media entre las dos metodologías de medición (el sesgo, es decir, cuánto se alejan las medidas del 0) y los límites de concordancia \pm 1.96 , con un nivel de confianza de 95\% (Costa-Santos et al. 2010).

La curva de supervivencia de la diferencia de las medias, desarrollada por Luiz et al. (2003) expresa el nivel de concordancia o discordancia como una función de tolerancia, utilizando el método de Kaplan-Meier (Kaplan y Meier, 1958), donde las fallas ocurren exactamente en valores absolutos de las diferencias entre los dos métodos. De este modo, en primer lugar, se calcula la diferencia absoluta de las medidas $|\mathrm{X}-\mathrm{Y}|$ y se representa en el eje de abscisas, mientras que en el eje de ordenadas se encuentra la proporción de los casos que tienen al menos la diferencia observada $x_{i}$. Este método mide la concordancia y su relación con la importancia de las diferencias observadas (Llorca y Delgado-Rodríguez, 2005). 


\section{Resultados y Discusión}

El análisis descriptivo de los datos de pesaje se presenta en el Cuadro 2. No se observan diferencias notables entre los dos tipos de medición del peso, donde la diferencia media entre los pesos es de $13.8 \mathrm{~kg}$. Los resultados con la balanza digital aparentan tener un peso medio mayor, mientras que con la cinta torácica se observa un mayor rango. Estas afirmaciones quedan constatadas en la Figura 1, donde además se destaca un desplazamiento de las cajas hacia la derecha, la ausencia de observaciones atípicas y la asimetría de los datos en los dos casos. Por otra parte, llama la atención que el peso obtenido con la balanza digital destaca no sólo por su media, sino también por tener un mínimo y mediana mayor que aquellas obtenidas mediante la cinta torácica.

El CCC produjo un resultado de 0.6961 , con un intervalo de confianza de $\mathrm{IC}_{0.95}=$ (0.5314 - 0.8102), lo cual, según el Cuadro 1 , corresponde a un nivel de concordancia entre los métodos de pesaje de «pobre». En la Figura 2 se observa la dispersión de los pesos obtenidos con ambas herramientas de medida, incluyendo la diagonal de concordancia. Concretamente, se percibe que los puntos están dispersos de forma aleatoria; es decir, sin presentar un patrón definido y distantes de la diagonal, concentrándose en el cuadrante inferior izquierdo. Por otra parte, el gráfico refleja que existe una relación positiva entre las dos variables. Por consiguiente, por medio del CCC se puede afirmar que ambas metodologías no son intercambiables, ya que no poseen una concordancia acreditable.

A través del procedimiento descrito por Bland y Altman, se obtuvo el gráfico mostrado en la Figura 3, donde se aprecia que la diferencia de las medias fue de 16.78 y que la diferencia entre los dos métodos posee una cierta tendencia a disminuir conforme aumen$\tan$ los valores medios. Esta tendencia decreciente se ve acentuada a partir de los 436
Cuadro 2. Estadística descriptiva de los pesos $(\mathrm{kg})$ de los bovinos según el método de pesaje $(n=46)$

\begin{tabular}{lcc}
\hline Método de Pesaje & $\begin{array}{c}\text { Balanza } \\
\text { digital }\end{array}$ & $\begin{array}{c}\text { Cinta } \\
\text { torácica }\end{array}$ \\
\hline Mínimo & 351 & 326 \\
Máximo & 524 & 531 \\
Cuartil inferior & 379.2 & 356.5 \\
Mediana & 408 & 387 \\
Cuartil superior & 442.8 & 425.2 \\
Media & 411.5 & 394.7 \\
Desviación típica & 40.2 & 48.7 \\
CV, \% & 9.8 & 12.3 \\
\hline
\end{tabular}

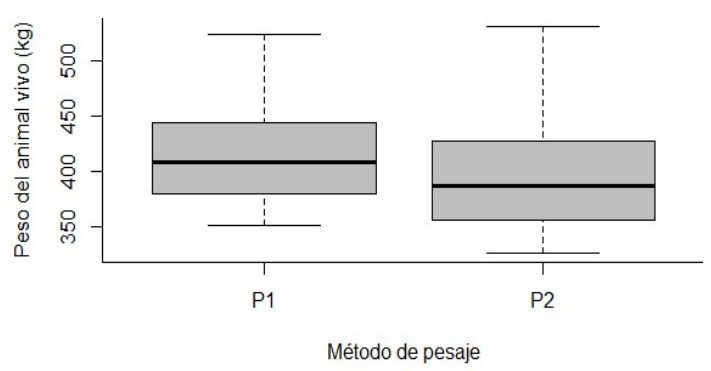

Figura 1. Distribución del peso vivo $(\mathrm{kg}) \mathrm{de}$ 46 Nelore machos medido a través de la balanza electrónica digital (P1) y la cinta métrica torácica $(\mathrm{P} 2)$

$\mathrm{kg}$ de media. Los límites de concordancia fueron de $[-45,43 ; 79,10]$ y la dispersión de los datos parece ser uniforme y aleatoria. El sesgo es significativo, a excepción de un punto, ya que el resto están comprendidos dentro de los límites de concordancia. De la misma manera, como el $95 \%$ de los puntos está comprendido dentro de los límites, podría apartemente concluirse que la concordancia entre los dos métodos es aceptable. Sin embargo, se reconoce que a mayor amplitud de los límites es menor la concordancia. En este caso, la amplitud de los límites es de 124.53, lo que explica que la mayor parte de los puntos esté comprendida dentro de estos $y$, que 


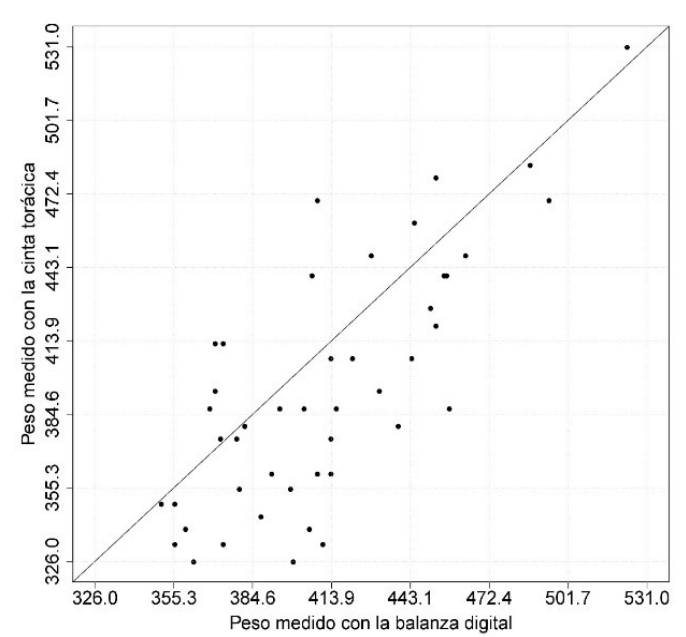

Figura 2. Diagrama de dispersión del peso (en kg) obtenido con la balanza digital frente al peso medido con la cinta torácica, incluyendo la diagonal de concordancia

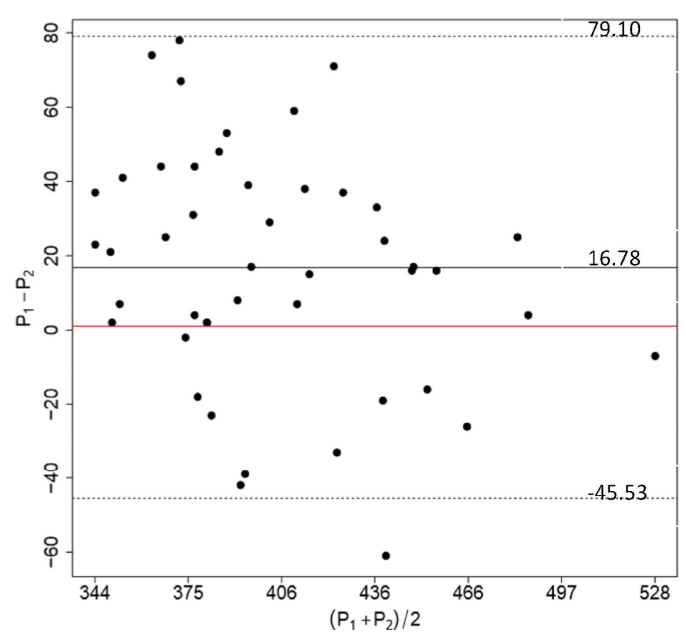

Figura 3. Gráfico de Bland-Altman del peso (en $\mathrm{kg}$ ) obtenido mediante la balanza digital (P1) y la cinta torácica (P2), incluyendo los límites de concordancia \pm 1.96 y la diferencia de las medias

a su vez, es un indicativo de que la balanza digital y la cinta torácica no son concordantes.

En la Curva de Supervivencia de la diferencia de las medias (Figura 4) se aprecia que la proporción va disminuyendo a medida

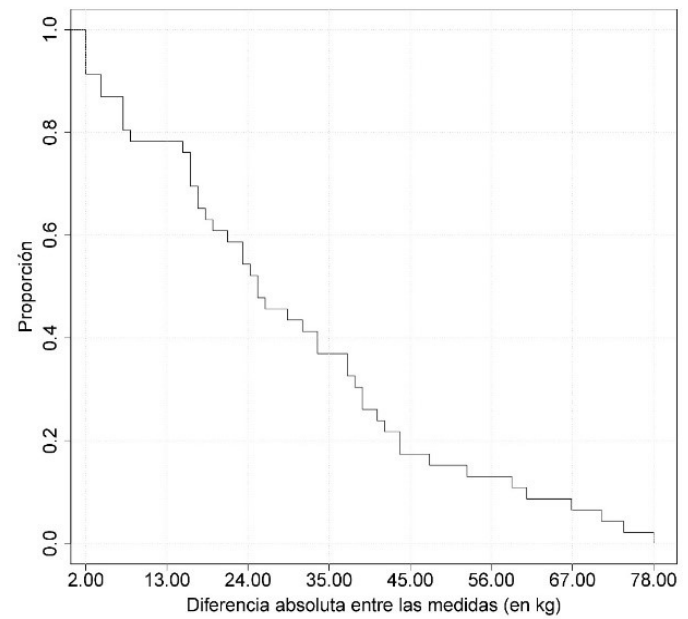

Figura 4. Curva de concordancia-supervivencia de la diferencia absoluta entre las medidas del peso (en $\mathrm{kg}$ ) obtenidas mediante la balanza digital y la cinta torácica (|P1-P2|) frente a la proporción del total de la unidad muestral que representan

que la diferencia va aumentando. Asimismo, llama la atención que todas las medidas poseen una diferencia de al menos $2 \mathrm{~kg}$ y que el $50 \%$ de los datos tiene una diferencia igual o superior a los $24 \mathrm{~kg}$. Sin duda, las diferencias que presentan los datos son un indicativo de que la concordancia no es demostrable, y que los resultados obtenidos con la cinta torácica no tienen equivalencia con las mediciones realizadas con la balanza digital.

La aplicación de la prueba $t$ de Student para muestras independientes utilizado por Watanabe et al. (2017) con estos mismos datos no rechaza la hipótesis de igualdad entre las medias, dado que las medidas fueron realizadas en la misma muestra, afirmando que las variaciones en el peso de los animales no eran significativas si se comparan los dos métodos de pesaje. Sin embargo, el presente estudio verifica, por medio de los tres métodos aplicados, que las medidas realizadas con la balanza digital y la cinta torácica presentan diferencias, lo que hace imposible que sean intercambiables y, por lo tanto, pueden acarrear mediciones erróneas. 


\section{ConClusiones}

- Los análisis llevados a cabo con el Coeficiente de Correlación de Concordancia de Lin, el Gráfico de Bland-Altman y la Curva de Concordancia-Supervivencia indican que el peso de bovinos obtenido con una balanza digital y con una cinta torácica no son concordantes para los datos del peso de novillos Nelore.

- Las medidas presentan diferencias que imposibilitan que estos métodos sean intercambiables.

- El cálculo del peso de bovinos Nelore mediante la cinta torácica proporciona medidas que pueden llevar a conclusiones erróneas.

\section{Literatura Citada}

1. Bland JM., Altman DG. 1999. Measuring agreement in method comparison studies. Stat Methods Med Res 8: 135-160. doi: 10.1177/ 096228029900800204

2. Costa-Santos C, Antunes L, Souto A, Bernardes J. 2010. Assessment of disagreement: a new information-based approach. Ann Epidemiol 20: 554-561. doi: 10.1016/j.annepidem.2010.02.011

3. da Silva DZ, Machado DM, Azevêdo $A$, Guimarães JE, de Oliveira ME, Mendes CE. 2006. Estimativa do peso vivo através do perímetro torácico de ovinos Santa Inês. Rev Cient Prod Anim 8: 41-46. doi: 10.15528/389

4. Garro JM, Rosales LR. 1996. Relación entre el peso corporal y el perímetro torácico en ganado cebuino en crecimiento en Costa Rica. Agron Costarric 20: 113-123.

5. Heinrichs AJ, Hargrove GL. 1987. Standards of weight and height for Holstein heifers. J Dairy Sci 70: 652-660. doi: 10.3168/jds.S0022-0302(87)80055-3
6. Heinrichs AJ, Rogers GW, Cooper JB. 1992. Predicting body weight and wither height in Holstein heifers using body measurements. J Dairy Sci 75: 3576-3581. doi: 10.3168/jds.S0022-0302(92)-78134-X

7. Kaplan EL, Meier P. 1958. Nonparametric estimation from incomplete observations. J Am Stat Assoc 53: 457481. doi: $10.2307 / 2281868$

8. Kashoma IPB, Luziga C, Werema C W, Shirima G A, Ndossi D. 2011. Predicting body weight of Tanzania shorthorn zebu cattle using heart girth measurements. Livestock Res Rural Dev 23(4). [Internet]. Disponible en: http:// www.lrrd.org/lrrd23/4/kash23094.htm

9. Lesosky M, Dumas S, Conradie I, Handel IG, Jennings A, Thumbi S, Toye $P$, et al. 2013. A live weight-heart girth relationship for accurate dosing of East African Shorthorn zebu cattle. Trop Anim Health Pro 45: 311-316. doi: 10.1007/s11250-012-0220-3

10. Llorca J, Delgado-Rodríguez M. 2005. Survival analytical techniques were used to assess agreement of a quantitative variable. J Clin Epidemiol 58: 314-315. doi: 10.1016/j.jclinepi.2004. 10.011

11. Luiz RR, Costa AJ, Kale PL, Werneck GL. 2003. Assessment of agreement of a quantitative variable: a new graphical approach. J Clin Epidemiol 56: 963-967. doi: 10.1016/S0895-4356(03)00164-1

12. Mahecha L, Angulo J, Manrique, LP. 2002. Predicción del peso vivo a través del perímetro torácico en la raza bovina $\mathrm{Lu}-$ cerna. Rev Colomb Cienc Pec 15: 88-91.

13. McBride GB. 2005. A proposal for strength-of-agreement criteria for Lin's Concordance Correlation Coefficient. NIWA Client Report 45: 307-310.

14. Mejia N, Velázquez C, Tewolde A. 1996. Predicción del peso vivo en ganado bovino, a partir de mediciones corporales. Agronomia Mesoamericana 7: 73-76. 
15. Ozkaya S, Bozkurt Y. 2009. The accuracy of prediction of body weight from body measurements in beef cattle. Arch Tierzucht 52: 371-377.

16. Reis L, Rodrigues MA, Dourado FH, Araújo B, Teodoro G, Dias RL, et al. 2008. Predição do peso vivo a partir de medidas corporais em animais mestiços Holandês/Gir. Cienc Rural 38: 778-783. doi: 10.1590/S0103-84782008000300029

17. Sánchez R. 2015. T-Student. Usos y abusos. Rev Mex Cardiol 26: 59-61.

18. Setim DH, Vanzan M, Fernandes MV, Metz P. 2010. Comprovação da eficácia do uso da fita torácica de pesagem em bovinos leiteiros. Instituto Federal Farroupilha. [Internet]. Disponible en: http://www.cafw.ufsm.br/ mostraciencias/2011/resumos/210.pdf
19. [USDA] Departamento de Agricultura de los Estados Unidos. 2017. Artículo en revista electrónica Economia em Dia: Carne bovina [Internet], [9 junio 2017]. Disponible en: http://usda.mannlib.cornell.edu/usda/fas/livestock-poultry$\mathrm{ma} / / 2010 \mathrm{~s} / 2016 /$ livestock-poultry-ma10-12-2016.pdf

20. Watanabe AH, Manchini H, Sachetin W. 2017. Comparação do peso corporal obtido através de pesagem em balança digital ou fita torácica de pesagem em bovinos Nelore. Pubvet 11:527-531

21. Yan T, Mayne CS, Patterson DS, Agnew RE. 2009. Prediction of body weight and empty body composition using body size measurements in lactating dairy cows. Livest Sci 124: 233-241. doi: 10.1016/j.livsci.2009.02.003 